\title{
Orden de los argumentos en enlhet
}

\section{Notas de introducción}

El enlhet, que es hablado en el Chaco Central paraguayo, es con aproximadamente 8.200 personas la lengua con la mayor cantidad de hablantes de la familia lingüística enlhet-enenlhet (anteriormente maskoy), a la que pertenecen también el enxet, el angaité, el sanapaná, el guaná y el toba-enenlhet (Unruh y Kalisch 2003, Fabre 2005).

Como base de la siguiente discusión, se resumen en adelante dos características de la sintaxis enlhet. Primero, las posiciones argumentales que el predicado verbal abre se saturan referencialmente dentro del mismo; dicho de otra manera, en el predicado verbal se establece una referencia a cada uno de los participantes involucrados en el estado de cosas expresado por el verbo. ${ }^{1}$ Por lo tanto, no es necesario realizar sintácticamente los argumentos verbales, sea a través de pronombres, demostrativos o material lexical que nombre los respectivos participantes. Es decir, el verbo en sí es suficiente para expresar una proposición completa (Kalisch 2009-2010); véase (1b). Para reflejar terminológicamente que no todo argumento está realizado como constituyente sintáctico, hago la siguiente diferenciación: el término argumento indica una posición requerida por la estructura lexical del verbo, el de participante un referente discursivo que ocupa cierta posición argumental, y el de expresión de participante un argumento que está realizado como constituyente sintáctico. ${ }^{2}$

\footnotetext{
1 Referencia, tal como uso el término, es el acto de identificar una entidad mental, el referente discursivo (Karttunen 1976); uso, abreviando, los términos de "referencia” y "referente" en vez de "referencia discursiva" y "referente discursivo".

2 Los enunciados enlhet en este texto forman parte de una recopilación de relatos orales realizada en el marco de Nengvaanemkeskama Nempayvaam Enlhet (www.enlhet.org) en las últimas dos décadas, que se complementa con un registro de expresiones que provienen de situaciones comunicativas variadas (en cuyo caso no se menciona el autor). El análisis aquí presentado se basa en este cuerpo de expresiones, y los enunciados son ejemplos representativos de las constelaciones descritas. Representan siempre expresiones continuas; los incisos se usan para facilitar la referencia a las partes de una misma expresión.
}

Hannes Kalisch, Nengvaanemkeskama Nempayvaam Enlhet, Filadelfia (359), Paraguay

Ә Open Access. (C 2019 Hannes Kalisch, published by De Gruyter. (cc) BY-NC-ND This work is licensed under a Creative Commons Attribution-NonCommercial-NoDerivatives 4.0 International License.

https://doi.org/10.1515/9783110605679-007 
(1) a. $\quad$-o- $-\mathrm{km}-\mathrm{o}$

lhnek ma' ak-nengken-a-klha haapen'. ${ }^{3}$

llegar-FACT-IBI-SUBJ PP:CIT DEM:DL F-poner:DIST-SUBJ-ESP palo.cruz:F [Se habla de un niño y su abuela]. 'Llegó a donde ella había dejado los palos de palo cruz.'

b. K-elyaky-aas-kek nek. Pk-enengk-aas-ee-nt-aak nek. F/M-elegir-CAUS:L-PRIM PP:CIT M-correr-CAUS-FACT-VENT-PRIM PP:CIT '[Él] los revisó y trajo uno.'

(Metyeeyam', 4/2/2001)

Segundo, la sintaxis enlhet no ofrece ningún sistema formal para marcar relaciones sintácticas o semánticas entre las expresiones de participante y el predicado verbal, la expresión de evento (información sobre cuál es, por ejemplo, sujeto y objeto o agens y patiens). ${ }^{4}$ Entonces, aunque el verbo marca el género del primer argumento (o sujeto), en un caso como (2) y (3), donde ambos participantes tienen el mismo género, no hay ningún criterio formal que indique la relación gramatical entre ellos.

Ang-ya'pa-s-kas-kek lhaak sa'kok meeme.
F-bañarse-CAUS:L-CAUS-PRIM PP:recién niña 1SG:madre
'Mamá bañó a la niña.' / 'La niña bañó a mamá.'

(3)
Ang-ya'pa-s-kas-kek
lhaak meeme
sa'kok.
F-bañarse-CAUS:L-CAUS-PRIM PP:recién 1SG:madre niña
'Mamá bañó a la niña.' / 'La niña bañó a mamá.'

\begin{abstract}
3 : separador de dos categorías dentro de un mismo morfema; ; separador de lecturas de un morfema; 1 primera persona; 2 segunda persona; 3 tercera persona; ABI abitivo; APR aproximativo; BEN benefactivo; CAUS causativo; CIT reportativo; COMP complexivo; CONJ conjetura; COOR coordinación; CÓP cópula; DEM demostrativo/pronombre; DIST distributivo; DL distal; ENF enfático; ESP especificativo; EXT extensivo; F femenino (delante de una raíz indica actor o poseedor, detrás una raíz indica género); F/M femenino o masculino; FACT factivo; FRUST frustrativo; FUT futuro; IBI ibi-locativo; IM imperativo; IMP impersonal; IND indefinido; INF infinitivo; INJ interjección; INT intensivo; INTER interrogación; INV inverso; L uso lexicalizado del morfema; LOC locativo; M masculino (delante de una raíz indica actor o poseedor, detrás una raíz indica género); NEG negación; NO.FACT no factitivo; PAS pasado; PASIVO pasivo; PL plural; POS posesivo; POT potencial; PP partícula predicativa; PAS.REM pasado remoto (o indefinido); PRES presentativo; PRIM primativo; PRV perventivo; REP repetitivo; SEC secundivo; SG singular; SUBJ subjuntivo; VENT ventivo.

4 Para reflejar eso terminológicamente hablo del primer y segundo argumento (que corresponden grosso modo a "actor” y "undergoer”); por definición, el primer argumento es aquel que puede ser marcado con un prefijo personal no inverso.
\end{abstract}


Recalco explícitamente que la posición de las expresiones de participante en la cláusula no está aprovechada para marcar relaciones sintácticas o semánticas. Sin embargo, eso no significa que el orden de los las expresiones de participantes sea libre. Más bien, sirve para estructurar pragmáticamente las proposiciones dentro del discurso; o sea, para relacionar expresiones lingüísticas con situaciones comunicativas concretas. Corresponde a estrategias discursivas. En este sentido, (2) y (3) expresan una ligera diferencia de sentido que no se refleja en la traducción al castellano: en (2), meeme 'mi madre' es el referente sobre el cual se habla; en (3) lo es sa'kok 'niña'.

El referente sobre el cual se habla (el referente sobre el cual la proposición correspondiente dice algo) está en una relación de tópico con esta proposición. ${ }^{5}$ Dicho de forma abreviada, es un tópico (en este párrafo sigo a Lambrecht 1994). Mientras que el tópico siempre forma parte de la presuposición, la pieza de información nueva que no es predecible, o recuperable del contexto, a la hora de la expresión -aquella información que no está presupuesta- se encuentra en una relación de foco con la proposición. Las funciones pragmáticas de tópico y foco, que corresponden a la diferenciación de información presupuesta e información afirmada, dan forma a la estructura informativa de la cláusula.

Para estructurar la información, el enlhet emplea una distinción formal entre la cláusula con verbo inicial y otra con expresión de participante inicial (este texto se limita a la discusión de cláusulas con predicado verbal). En la sección 2 presentaré la cláusula con verbo inicial y resumiré los parámetros que rigen tanto el posicionamiento de las expresiones de participante dentro de la misma como la opción de no realizar sintácticamente cierto participante. En la sección 3 describiré la cláusula con expresión de participante inicial que se usa para expresar diferentes tipos de foco. En la sección 4 parto de la observación de que existe una superposición de las condiciones asertivas bajo las cuales se usan los dos tipos de cláusulas, para concluir que las funciones pragmáticas de tópico y foco no bastan para describir su respectivo empleo. Más bien, se vuelve necesario analizar también las situaciones comunicativas en las cuales se usan para determinar, con ello, las funciones discursivas que expresan más allá de las funciones pragmáticas.

5 Como proposición entiendo el estado de cosas expresado a través de una cláusula. 


\section{La cláusula con predicado verbal inicial}

En la construcción más frecuente, el predicado verbal (la expresión de evento) aparece en la posición inicial de la cláusula y las expresiones de participante le siguen. Ahora bien, el orden de las expresiones de participante no puede discutirse de forma independiente de los parámetros que requieren, o posibilitan, la realización sintáctica de los argumentos verbales. A tal realización sintáctica de un argumento a través de una expresión de participante que complementa su saturación referencial en el verbo me refiero en adelante con el término elaboración (de un participante).

Los parámetros que rigen tal elaboración son múltiples: la necesidad de introducir nuevos referentes discursivos, de indicar la estructura informativa de la cláusula, de brindar información lexical, de desambiguar la expresión, de reforzar relaciones de correferencia, de indicar aspectos semánticos como la genericidad y la especificidad, entre otros. Sin embargo, en el marco de la presente discusión esta multiplicidad de parámetros se vuelve manejable si determinamos aquellos que posibilitan la no-elaboración de un argumento, pues como veremos, tanto el posicionamiento de las expresiones de participante como la no-elaboración de un participante responden a requerimientos discursivos que tienen que ver con la $a c$ cesibilidad cognitiva del referente correspondiente (o sea, el conocimiento sobre el mismo) y su estado de activación mental (la conciencia de él en un momento discursivo dado; Lambrecht 1994, 93). En este sentido, en la sección 2.1 resumiré las condiciones bajo las cuales un argumento no está elaborado sintácticamente. En la sección 2.2 sintetizaré las estrategias para ordenar las expresiones de participante cuando una cláusula tiene más que una.

\subsection{El argumento no realizado sintácticamente}

He señalado que la saturación referencial del argumento verbal se produce dentro del mismo verbo; esta saturación inherente permite la correferencia de una manera comparable a lo que hacen los pronombres en lenguas como el inglés o el castellano. Por lo tanto, similar a lo que ocurre con el sujeto en castellano, bajo ciertas circunstancias un argumento no está realizado como constituyente sintáctico (véase (4d)); o, alternativamente: cierto participante no está elaborado. Tal no-elaboración de un participante es posible siempre que se trate de un referente activado que es, a la vez, tópico (los parámetros que rigen la realización sintáctica de un tópico, en cambio, no son discutidos aquí). Dicho a la inversa, la no-elaboración de un participante implica una lectura como tópico. Esta sería, así, la lectura básica de los argumentos inherentemente saturados. 
El ejemplo de (4) sirve para ejemplificar la no-elaboración de un participante. En (4a) se habla sobre una pareja, una mujer y su esposo. La proposición de (4b), en cambio, es solo sobre el hombre, quien es el tópico (la referencia a un participante activado puede ser reforzada explícitamente a través del demostrativo/pronombre ma'a ma' que indica en un sentido muy amplio accesibilidad). En (4c), se vuelve a hablar de la mujer, pero esta está relativamente menos activada que el hombre; por lo tanto, está representada a través de material lexical, aptaava' 'su esposa' (y no es tópico), mientras que la proposición es nuevamente sobre el hombre, que no está elaborado. Sin embargo, una vez mencionada la mujer, ambos referentes tienen el mismo grado de activación y en (4d) ninguno de los dos está realizado sintácticamente. No hay criterios formales ni semánticos para decidir cuál de los dos es el tópico; la cláusula tiene dos tópicos. En (4e), en cambio, la mujer sigue siendo no elaborada, pero el hombre está representado a través de un demostrativo/pronombre. Con ello está relegado a una posición de no-tópico, como veremos en la sección 2.2.

\section{a. “E-nelht-ee-nt-a'! iA-m-ok saat takha'”, M-saltar-POT-VENT-IM 1SG-agarrar-POT PP:FUT recepción:F}

[Se habla de una pareja; el hombre pica miel en la altura de un árbol y su mujer lo observa desde abajo.] ““¡Salta! ¡Te voy a atajar!”,

nek maata' an-t-eem-ak kelvaana'a.

PP:CIT PP:siempre F-CÓP-IND-SEC mujer:F

'repetía la mujer.'

Sellook ne pk-aasekh-ay'-ook

después.de.un.tiempo PP:CIT M-atender;respetar-IND-INT

ma'a, a-taava'.

DEM:DL F esposo:M/F

'Después de un tiempo, él le hizo caso, su esposo.'

b. Taa' ap-nelht-ayke-nt-amo lhnek ma'a, ipokj!

después M-saltar-IND-VENT-SUBJ PP:CIT DEM:DL INJ

'Después, [él] saltó hacia abajo [del árbol a la tierra].'

c. Am-pathet-m-eek nek ma' ap-taava', F-agarrar-IND-PRIM PP:CIT DEM:DL M-esposo:M/F

'Su esposa lo agarró,'

d. k-el-vaapokh-eeyk-am', an-t-om-ke';

F/M-DIST-torcer-IND-EXT F-comer-IND-PRIM

'le torció [el cuello], lo comió;'

e. savh-om-ook nek ma'a.

terminar-IND-INT PP:CIT DEM:DL

'acabó completamente con él.' (Seepe-Pta’heem-Pelhkapok, 6/2/2005) 
Ahora bien, en las cláusulas de (4d), en las cuales ninguno de los participantes está elaborado, hay un paralelismo entre el referente discursivo y su relación lógica o semántica con el evento correspondiente: en ambos casos, la mujer corresponde al primer argumento y el hombre al segundo. Por lo tanto, se podría sospechar una correlación sistemática entre el tópico y el primer argumento (o sujeto) de verbos bivalentes como se conoce del castellano (Hockett 1958, 201, Ashby y Bentivoglio 1993) y cuestionar, así, la existencia de dos tópicos. Sin embargo, en (5) vemos que la correlación del tópico movheeva 'víbora' con el primer o el segundo argumento varía repetidas veces dentro de un mismo párrafo. En (5a) se introduce un nuevo referente (ver sección 2.3), movheeva 'víbora'; como pieza nueva de información (o sea, como información que no está presupuesta) está en foco. Una vez introducida, sin embargo, funciona como el tópico de las cláusulas siguientes y no está elaborada en las cláusulas siguientes porque es tópico. Ahora bien, en (5b) y (5c), movheeva 'víbora' corresponde al segundo argumento, a la vez que el primer argumento equivale - primero- en (5b) al yo narrador, que no está elaborado y figura como un segundo tópico (no importa aquí el comportamiento especial de la referencia al hablante en la sintaxis enlhet); y -segundo- en (5c) a semheng 'perro' (que, como referente nuevo, está en foco). En (5d) y (5e), movheeva 'víbora' vuelve a corresponder al primer argumento, en (5f) al segundo, en (5g) nuevamente al primero, y en (5h) al segundo. Se ve, en fin, que un referente no-elaborado puede corresponder a diferentes posiciones argumentales en cláusulas subsiguientes sin que eso requiera una aclaración con medios formales. Como señalado, un participante no elaborado siempre es tópico; por ende, no existe una correlación entre el tópico y el primer argumento y se afirma que en cláusulas como (4d) o (5b), (5e) o (5f) hay dos tópicos.

(5) a. Movheeva k-vesey' a, a-l-yelhvaas-k-aok nak tipo.de.víbora:F F-nombre:F DEM:DL F-DIST-rojo-IND-COMP PP:PRES a.

DEM:DL

'Movheeva se llama, aquella con rayas rojas.'

b. Naate k'o k-tahany-a'ak sek-tey-aas-k-am' alhta'a, PP:PAS.REM 1SG 1SG-encontrar-SEC 1SG-andar-CAUS:L-IND-EXT noche:F 'Con esta me encontré cuando andaba en la noche,'

ya'alva s-tengy-ey', lenteenoo nhan. tatú:M 1SG-conseguir-IND linterna:F PP:COOR "buscando armadillos con una linterna.' 
c. Hayyy, ya'm-as-a m'a semheng, INJ perseguir-CAUS:L-SUBJ DEM:DL perro:F

'El perro la perseguía,'

d. mey-a-nt-aak maata'a va'lhok e-yaay-a'aok, ir-FACT-VENT-PRIM PP:siempre interior:F 1SG-pierna-PL 'y ella entraba entre mis pies,' yapop-kek naate yka', cegar-PRIM PP:PAS.REM PP:pero 'pero estaba cegada [por la linterna],'

e. hoo' me-he-taklh-am-a. por.eso NEG-INV.SG-morder-IND-SUBJ 'por eso no me mordió.'

f. Ay-engk-ee nyek maata'a savov, 1SG-cortar-PRIM PP:FRUST PP:siempre cuchillo:F 'Yo intentaba cortarla con el machete,' pokt-ae-km-ek maata'a, llegar;tarde-IND-APR:L-PRIM PP:siempre 'pero siempre llegaba tarde,'

g. mey-a-klh-eek maataa' a, ir-FACT-REP-PRIM PP:siempre DEM:DL 'se iba siempre al otro lado,'

h. ay-engk-ee nyek maata'a savov, paek. 1SG-cortar-PRIM PP:FRUST PP:siempre cuchillo:F INJ:no 'intentaba cortarla con el machete, jno!'

Lheema k-peyv-aa-mk-o-ek sey-engk-a naa-lhet. por.fin 1SG-acertar-FACT-COMP-INT-NO.FACT 1SG-cortar-SUBJ F-mitad:F 'Al final tuve suerte y la corté en el medio.'

i. ¡Paaat ay-eny-eem ma'a, nemesma nak ma'a! rápido:ENF F-correr;huir-IND DEM:DL víbora:F PP:PRES DEM:DL ‘¿Esa víbora es rápida!’

(Metyeeyam', 7/2003)

Es necesario añadir una nota sobre la realización sintáctica de un no-tópico. En (6a) se introduce un nuevo referente, valay 'paraguayo', que no está presupuesto; o sea, que está en foco. A partir de (6b), este referente es tópico (por lo que deja de estar elaborado), y en (6c) se introduce otro referente que no está presupuesto, taata 'mi padre', que está, así, nuevamente en foco. Es decir, si bien he mencionado que la lectura básica de los argumentos inherentemente saturados es como tópico, su realización sintáctica concreta puede cancelar o reafirmar esta lectura: en (6c), por ejemplo, taata 'mi 
padre', al ser una pieza nueva de información, está en foco y cancela dicha lectura básica, mientras que el tópico ma'a valay 'los paraguayos' la reafirma. En síntesis, la cláusula con verbo inicial tiene sistemáticamente más de una lectura en lo que respecta a su estructuración pragmática; en otras palabras, es vaga con relación a la diferenciación entre información presupuesta e información afirmada. En la sección 4, esta observación será de importancia.

(6) a. Na-taamen ang-yaanmaan apk-el-v-a'a-kt-amo

F-detrás F-iniciación:F M-DIST-llegar;buscar-FACT-VENT-SUBJ

valay.

paraguayo

'Después de la fiesta vinieron paraguayos.'

b. A-peen-aas-kek apk-el-laap-omm-aap a, M-terminar-CAUS:L-PRIM M-DIST:L-matar-IND-PAS:M DEM:DL

'Ellos habían terminado su batalla'

taan ap-v-a'a-kt-amo hay' tengma,

después M-llegar;buscar-FACT-VENT-SUBJ DEM:aquí casa;aldea:M

'y vinieron a nuestra aldea'

mah-ay' heng-el-laap-ok. (...)

querer-IND INV:PL-DIST:L-matar-POT

'para matarnos. (...)'

c. Apk-el-yoot-ey'-aa yke la

M-DIST:L-tirar-IND-PRIM PP:pero PP:duda

k'o taata m'a valay, paej eeka'.

1SG 1SG:padre DEM:DL paraguayo NEG PP:pero

'Los paraguayos tiraron [con sus fusiles] a mi padre, pero no [pasó nada].'

(Sa'kok-Nay', 28/7/2009)

\subsection{El orden de las expresiones de participante}

En (6c) vemos una cláusula con más de una expresión de participante. Para describir el orden de estas expresiones de participante, la escala (7) cobra importancia (Chafe 1987, 25ss., Lambrecht 1994, 93ss.). Esta escala conjuga el grado de accesibilidad cognitiva de un referente - saber de él- con su estado de activación mental - pensar en él en un momento dado-. 
(7) estados pragmáticos de los participantes: inactivado < accesible < activado

Existe una relación entre el estado pragmático de un participante y su función pragmática, pues cuanto más alto un participante está en la escala (7), más aceptable es como tópico (Lambrecht 1994: 165). Al mismo tiempo, sin embargo, estar activado no es una característica exclusiva del tópico; un participante en foco puede estarlo también. Dado, entonces, que la posición sintáctica de un foco difiere de la de un tópico, una descripción de la posición preferencial de las expresiones de participante en la cláusula debe atender, aparte del estado pragmático, la función pragmática del participante correspondiente. Eso se refleja en la escala (8), la cual permite, además, describir las condiciones que rigen la noelaboración de un participante. Se lee de la siguiente manera: cuanto más alto está un referente en esta escala, menos prominente es su ubicación con relación al predicado verbal; o sea, aparece en la posición más alejada del mismo o no está elaborado directamente.

(8) preferencia de un participante de ocupar la posición menos prominente de la cláusula: inactivado < accesible < activado < tópico

Por ejemplo, en (4c) se realiza sintácticamente un referente activado que no es tópico, aptaava' 'su esposa', mientras que el tópico, el hombre, no está elaborado. En (6c), el referente accesible taata 'mi padre' aparece delante del referente valay 'paraguayo' que está activado porque aparece en las cláusulas inmediatamente precedentes (y que es, además, tópico). En (9a), se habla de enlhet-neeten 'ángel' y yaame'enlhet-neeten 'pseudo-ángel'; en (9b), ambos están activados y son además tópicos, razón por la cual no están elaborados. En (9c), el tópico yaame'enlhet-neeten 'pseudo-ángel' se posiciona detrás del referente no-específico enlhet 'hombre' que está inactivado, o sea, no es recuperable del contexto. En (9e), enlhet-neeten 'ángel' y yaame'enlhet-neeten 'pseudo-ángel' no están elaborados, porque son tópicos, mientras que el demostrativo/pronombre ma'a crea una relación anafórica con enlhet 'hombre' que fue mencionado en (9c); o sea, aunque está activado, no es tópico (compárese con (4e)).

(9) a. Taan ko'o se-nengkoongn-engv-ay'-a yaame'-enlhet-neeten. después 1SG INV:SG-ir.debajo.de-LOK-IND-SUBJ como-enlhet-arriba 'Después se juntaron pseudo-ángeles conmigo.'

Yaam-kelhvook se-nengkoongn-engv-ay'-a nhan lha como-en.seguida INV:SG-ir.debajo.de-LOK-IND-SUBJ PP:COOR DEM:2 
enlhet-neeten, payh-ook a Peetempok.

enlhet-arriba estar.cerca-INT DEM:DL Neu-Halbstadt

'Hace relativamente poco tiempo se me acercaron también algunos ángeles [de los que hablamos], allí en los alrededores de Neu-Halbstadt.'

b. Haave' apk-el-a'ank-amm-aap.

no.es M-DIST-rechazar-IND-PASIVO:M

'No se rechazan mutuamente.'

c. Vaamlha' apk-avn-a nak enlhet a solo M-encantar-SUBJ PP:PRES enlhet DEM:DL

yaame'-enlhet-neeten, como-enlhet-arriba

'Solo cuando un pseudo-ángel encanta a un hombre,'

d. taan ko'o angv-aekm-aks-ek ap-menaekm-aas-k-am' después 1SG 1SG-imitar-CAUS:L-POT M-cantar-CAUS:L-IND-EXT enlhet-neeten, enlhet-arriba 'entonces yo imito el canto de los ángeles,'

e. yo'yaam' saat e-yenyav-s-ek ma'a. para PP:FUT M-tirar-BEN-POT DEM:DL 'para que ellos lo separen de aquél.' ～(Maangvayaam'ay', 7/2/2017)

Esta observación relativa al demostrativo remite a la escala (10), a través de la cual describo la probabilidad con la cual las tres opciones básicas para expresar un participante (a través de material lexical, un demostrativo/pronombre o la no-elaboración) representan un tópico: pueden representarlo siempre que no aparezca en la misma cláusula otra opción que esté ubicada en una posición más alta de dicha escala. Es decir, mientras que el participante no-elaborado siempre es tópico, el demostrativo con función anafórica lo es ante la ausencia de un participante no-elaborado (véanse (4b), (4d), (5h) y otros más). Un referente realizado con material lexical, a su vez, puede ser tópico ante la ausencia tanto de un demostrativo/pronombre como de un participante no-elaborado (véase valay 'paraguayo' de (6c)).

(10) probabilidad de ser tópico: material lexical < demostrativo con función anafórica < no-elaborado

En (11b) se neutralizan las escalas (8) y (10), porque ambos participantes se realizan de la misma manera con material lexical, son inactivados (lo que significa 
que no son recuperables del contexto) y ninguno es tópico. Aunque el referente que ocupa en (11b) la última posición de la cláusula, lhoop'aktek 'tierra', coincide casualmente con el segundo argumento, el orden aquí no está impuesto por la jerarquía argumental. Más bien, se debe a que la tierra constituye el referente sobre el cual es la cláusula siguiente (11c). Si (11c) tratara sobre los vecinos, serían ellos los que deberían ocupar la posición final de (11b). Es decir, es posible expresar una intención pragmática, tratando un participante como si fuera aquel sobre el cual es la proposición -como tópico- (lo que significa que está relegado a la última posición de la cláusula), aunque las constelaciones asertivas lo identifican como pieza de información nueva, o sea como foco.

(11) a. Ka laa lha' ak-t-eem-a
cómo PP:duda PP:CONJ F-CóP-IND-SUBJ
me-heng-aap-eeyk-am-ka'a nak lengko.
NEG-INV:PL-atender-IND-EXT-SUBJ PP:PRES menonita
'¿Cuál podría ser la razón por la cual los menonitas no nos atienden?'
b. K-et-lheen-kek ko'o maata'a sek-moolhama
F/M-DIST-decir-PRIM 1SG PP:siempre 1SG-vecino
lhoop'aktek ap-angk-a'aok.
tierra:F M-POS-PL
'Mis vecinos suelen hablar de sus tierras [perdidas].'
c. Na'sook nahan lhoop'aktek eng-ak nengko'o,
de.verdad PP:COOR tierra:F 1PL-POS 1PL
ak-yav'aklhoho nak hay'.
F-todo;entero;en.cuerpo PP:PRES DEM:aquí
'Es cierto que todo por aquí es nuestra tierra.'

(Seepe-Pta'heem-Pelhkapok, 6/2/2005)

Comparando (9c) y (11b) vemos de nuevo que el posicionamiento de las expresiones de participante es independiente de su relación lógica o semántica con el evento correspondiente. Al igual que el tópico no-elaborado (véase (5)), el tópico elaborado puede coincidir bien con el primero, bien con el segundo argumento (o, en otra terminología, con el sujeto o el objeto). Sin embargo, en investigaciones futuras se debe aclarar si existen correlaciones significativas entre parámetros semánticos del argumento (por ejemplo, animado o no; propicio a ser agente o no; específico o no) por un lado y, por otro, el estado pragmático de un referente discursivo y la posición de la respectiva expresión de participante en la cláusula. Por ejemplo, el segundo argumento del verbo nentengyey' 'conseguir' (5b) siempre 
es no-específico y se deberían ver las consecuencias que tiene ello para su habilidad de ser el tópico de la cláusula y, por ende, para su posición sintáctica dentro de la misma.

\section{La cláusula con constituyente preverbal}

El enlhet ofrece una alternativa formal importante a la cláusula con verbo inicial, en la cual aparece una expresión de participante delante del predicado verbal ((12b), (12c)); hablaré en adelante de la cláusula con constituyente preverbal. El constituyente preverbal interactúa formalmente con el predicado verbal y en 3.1 describo las diferencias formales que existen entre los predicados verbales en uno y otro tipo de cláusula. En 3.2 resumo las constelaciones asertivas bajo las cuales se emplea la cláusula con constituyente preverbal y en 3.3 propongo una primera clasificación de sus distintos usos.

(12) a. Ap-lhaan-v-a'a-kt-aa':

M-decir-LOC-FACT-VENT-PRIM

'Vino a informar:'

b. "Vaetke ng-matn-ak.

vaca:F F-morir.a.la.fuerza-SEC

“'Se mató una vaca.'

c. Kenmekpeyem apk-a'h-ak.” ayoreo M-matar-SEC

'Los ayoreo la mataron."'

(Haakok Metaykaok, 9/12/2010)

\subsection{La forma del verbo no-inicial}

En la cláusula con una expresión de participante delante del verbo aparecen dos diferencias formales importantes en comparación con la cláusula con verbo inicial (compárese (13b) con (13c)), las cuales afectan la posición sintáctica de la partícula predicativa y el empleo de una de las cinco categorías que conforman el paradigma de los estados de subordinación verbal. En la cláusula con verbo inicial, las partículas predicativas siguen al predicado verbal y el mismo marca el estado de subordinación 1 o primativo, el cual indica el estado no-subordinado. En cambio, en la cláusula con constituyente preverbal estas partículas preceden al verbo y el mismo expresa el 
estado de subordinación 2 o secundivo, el cual se emplea cuando una expresión de evento que es lógicamente independiente de otra no ocupa la posición inicial de la cláusula. ${ }^{6}$

(13) a. Neng-halhn-oo' ay-enmong- $a$. 1PL-escuchar-INT F-estallar-SUBJ 'Escuchamos como estalló algo.'

b. “iNen-tekyev-ke?" ak-tamh-ak nahan. 1PL-morir:DIST-PRIM 1SG-CÓP-SEC PP:COOR ““iAlguien fue asesinado!”, dije todavía.’

c. Valay nooke laa lha' ap-matn-ak. paraguayo PP:de.verdad PP:duda PP:CONJ M-morir.a.la.fuerza-SEC 'Y de hecho, un paraguayo fue asesinado.' (Emilio·ngken', 29/6/2002)

Las dos características formales - la posición de la partícula predicativa y la categoría del estado verbal- están relacionadas entre sí. La función de las partículas predicativas es modificar la predicatividad del constituyente sintáctico al que siguen: cuanto menos reducida es, más posibilidades hay para emplearlas en combinación con el mismo (Kalisch 2009-2010, 119). Ahora bien, detrás de un verbo en el estado de subordinación 2 o secundivo están prohibidas; en cambio, siguen al constituyente preverbal. Eso implica que el verbo en secundivo ha perdido (por lo menos una parte de) su predicatividad -es precisamente eso lo que señala la categoría del secundivoy la misma recae sobre la expresión de participante preverbal. En consecuencia, evito en adelante usar el término predicado verbal cuando me refiero a una cláusula con constituyente preverbal y uso el de expresión de evento.

Las constelaciones descritas indican que la cláusula (13c) es comparable con una oración hendida que se traduciría como Era un paraguayo el que fue asesinado. Sin embargo, la pérdida de predicatividad del verbo hace que la misma no sea ya transparente como oración hendida, a la vez que el constituyente preverbal tenga lecturas claramente definidas, sobre las cuales trataré en adelante.

6 En oposición al secundivo, el estado de subordinación 3 o subjuntivo indica una dependencia lógica o formal de un predicado verbal de otro evento $u$ otra proposición (véanse $(5 \mathrm{~b})$, (20a)). En este espacio, la oposición entre secundivo y subjuntivo no puede ser discutida. 


\subsection{Condiciones asertivas del constituyente preverbal}

Identifiquemos las condiciones asertivas bajo las cuales la expresión de participante aparece en la posición preverbal (en adelante, se subraya el constituyente preverbal en los enunciados). En (14b), el participante prepuesto no forma parte de la presuposición, mientras que la expresión de evento y el segundo participante sí. Es decir, con el constituyente preverbal se presenta un referente que está en una relación de foco con la proposición correspondiente; en otras palabras, el constituyente preverbal expresa un foco sobre el participante.

(14) a. So' alh la y-ent-as-a aktam

¿qué? PP:PAS PP:duda F-romperse-CAUS-SUBJ naranja.de.monte:F

ma'a ye;

DEM:DL INTER

‘Quién era que cortó [la rama de] la naranja de monte?'

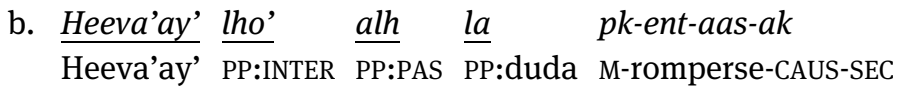

ma' aktam.

DEM:DL naranja.de.monte:F

'Creo que era Heeva'ay' quién cortó [la rama de] la naranja de monte.'

(Kaymaap-Maama, 29/6/2005)

Las constelaciones en (12b), en cambio, son diferentes, porque no se encuentra información presupuesta, o sea información recuperable del contexto. Es decir, el constituyente preverbal no es en sí el foco, sino forma parte de un foco que se extiende sobre toda la cláusula. Esta observación indica, primero, que el constituyente preverbal no es en sí el portador del foco. Más bien, es la forma de la cláusula entera que, en su oposición a la cláusula con verbo inicial, sirve para la codificación pragmática de la información. Segundo, hace necesario diferenciar el alcance del foco sobre los constituyentes sintácticos de la cláusula; o sea, requiere distinguir diferentes extensiones sintácticas del foco en las cláusulas con constituyente preverbal. Este es el objetivo de la sección 3.3.

\subsection{Las extensiones sintácticas del foco}

El foco expresado a través de la cláusula con constituyente preverbal se extiende a una expresión de participante o a la expresión de evento en combinación con una o más expresiones de participante. El foco sobre el predicado solo, en 
cambio, está expresado a través de una cláusula con verbo inicial como (15) y (18a), que no está sujeta a las siguientes observaciones.

(15) Ap-lheng-kek nek lhaak taata. M-viajar-PRIM PP:CIT PP:recién 1SG:padre '(Dicen que) papá se fue recién.'

(a) Foco sobre el participante o foco argumental (Lambrecht 1994, 222). En (16b), el evento forma parte de la presuposición y es el participante el que se encuentra en una relación de foco con la proposición; hablamos de un foco estrecho.

(16) a. “Ak-haave' enlhet, ¿sok laa lha' ap-s-oyk-am' F-no.es enlhet;humano ¿qué? PP:duda PP:CONJ M-llevar-IND-EXT ke?” “K-el-yenm-oo nak lhaak ma'a”, tamh-ak INTER F/M-DIST:LEX-brillar-INT PP:PRES PP:recién DEM:DL CÓP-SEC maata'a pook. PP:siempre otro:M “"No es hombre, ¿qué lleva consigo?” "Vi que brillaba”, dijo otro.'

b. "Nengmaskeem lho la $\underline{\text { la }}$ p-s-aakm-ok ma'a. enfermedad:F PP:INTER PP:duda M-llevar-APROX-SEC DEM:DL '“Parece que trae enfermedad.'

Yees-ook lhaa yempehek ma' ap-s-oyk-am' lhaak.” mojado-INT PP:recién F:piel:F DEM:DL M-llevar-IND-EXT PP:recién 'La superficie de lo que tiene consigo está mojada."'

(Savhongvay', 23/1/2002)

(b) Foco sobre la cláusula entera o foco oracional. En (17b), la cláusula no contiene información presupuesta. Es decir, no hay tópico, la pieza nueva de la información consiste en la expresión de participante y la expresión de evento en su conjunto y el foco coincide con la cláusula entera. Hablamos de una cláusula con foco amplio, en la cual ningún referente forma parte de la presuposición (Lambrecht 1994, 222).

(17) a. “¿Ko k-tamh-a p-n-ayk-am’”

cómo F-CÓP-SUBJ M-estar;sentarse-IND-EXT

““¿Cómo están?”,

b. “Lhama n-seka' ap-matng-ak!”

uno F-criatura M-morir.a.la.fuerza-SEC

““¡Un niño fue asesinado!”

(Maangvayaam'ay', 25/6/2015) 
El foco sobre la cláusula entera se aplica también a los verbos bivalentes (18b).

(18) a. “Tataa, itey-akm-ek yaamkentem'aahak ap-ak!” 1SG:padre caer-APR:L-PRIM bolsa:F M-POS

[Durante un viaje, alguien ve algo claro al lado del camino y dice:] “"Papá, ¡se cayó tu bolsa!”

b. Meyva yke nha' angk-a'h-ak yaata'ay'. "iMeyva!” puma:F PP:pero PP:COOR F-matar-SEC cabra:M puma:F 'En realidad, un puma había matado una cabra. "¡Es un puma!”'

(Kam'aatkok Ketsek, 5/8/2008)

Siendo, los dos, parte del foco oracional, los participantes meyva 'puma' y yaata'ay' 'cabra' tienen la misma condición pragmática, aunque ocupan distintas posiciones sintácticas. Como ejemplifica el par mínimo de (19b) y (20b), los participantes de esta índole pueden intercambiarse sin que se produzca ningún cambio de sentido pragmático. Ambos enunciados, que representan dos versiones de un mismo relato de un solo autor, muestran un foco oracional, pero en (19b) es el primer participante (o agente) el que ocupa la posición preverbal, mientras que en (20b) es el segundo participante (o paciente). En ambos casos, la cláusula subsiguiente es construida de forma completamente paralela; eso significa que la diferencia no puede explicarse con una intención pragmática como aquella mencionada con relación a (11b). Por alguna razón no-sistemática, el autor nombra a uno de los participantes primero, priorizándolo, así, de alguna manera.

(19) a. K-enengk-a-klh-o a tengma:

F/M-correr-FACT-REP-SUBJ DEM:DL casa;aldea:M

'Corrió a la aldea:'

b. “'Seenavakpo lhkaa ngk-en-naap-ak vaapa'ta'aok!” víbora.gigante:F PP:recién F-DIST:L-matar-SEC muchachos “" $\mathrm{i}$ Una víbora gigante mató a los muchachos!”'

c. “A, ta'se', jang-a'h-ek saat!”, ne pk-el-tamh-ak. INJ está.bien 1PL-matar-POT PP:FUT PP:CIT M-DIST-CÓP-SEC ““Está bien, ¡la vamos a matar!”, dijeron.'

(Maangvayaam'ay', 19/1/2017)

(20) a. Apk-enengk-eeyk-aoklh-o lhnek lhama m'a tengma: M-correr-IND-ABI-SUBJ PP:CIT uno DEM:DL casa;aldea:M 'Uno corrió a la aldea:' 
b. ¡Vaapa'ta'aok lhaa ngk-en-naap-ak nemesma! muchachos PP:recién F-DIST:L-matar-SEC víbora:F ““¡Una víbora mató a los muchachos!'

¡Tekyev-ke'!

morir.a.la.fuerza:DIST- PRIM

¡Murieron!””

c. "Nooka' a-ngkook ma'a, iang-en-naap-ok saat!” PP:de.verdad F-INT DEM:DL 1PL-DIST:L-matar-POT PP:FUT ““Está bien, ¡la vamos a matar!”’ (Maangvayaam’ay’, 20/12/2012)

(c) Foco compuesto. La cláusula con foco oracional no tiene tópico; en consecuencia, todos los participantes son realizados sintácticamente. ${ }^{7}$ Existe otro tipo de foco amplio, el foco compuesto, que está limitado a verbos bivalentes. En cláusulas con este foco, uno de los participantes es tópico y no está elaborado, mientras que el foco se extiende sobre la expresión de evento y el otro participante, que está realizado sintácticamente y puede coincidir tanto con el primer (21) como con el segundo argumento ((22) y (23)). Cabe añadir que, en términos formales, el foco compuesto y el oracional se diferencian por la cantidad de expresiones de participante en las respectivas cláusulas.

(21) Ap-veen-eek seepe', ang-ken' an-tekp-aak. M-llorar-PRIM niño F-madre F-pegar-SEC

'El niño llora, su madre le pegó.'

(22) $i V-a$ 'a-kt-aa

lyamhap-m-a! iLhaapen apk-a'h-ak!

llegar;buscar-FACT-VENT-PRIM casarse-IND-INF ñandú:M M-matar-SEC

‘¡Volvió el recién casado! ¡Cazó un ñandú! ～(Kooneng-Pa’at, 23/1/2007)

(23) A-petses-v-ee-klh-o

po-phehek, k-elpaats-ee-nt-amo

M-chupar-LOC-FACT-PRV-SUBJ M-dedo:F F/M-escupir-FACT-VENT-SUBJ

eema,

sangre:F

'Chupó su dedo y escupió sangre.'

inengmaskeem nooke laa lha' ap-tep-kesk-am-ak!

enfermedad:F PP:de.verdad PP:duda PP:CONJ M-salir-CAUS-IND-SEC ‘Había sido que [con este gesto] desató la viruela!'

(Metyeeyam', 24/5/2002)

7 Esta afirmación debe ser precisada para incluir el caso de que uno de los participantes refiera a la primera o segunda persona, para cuya realización sintáctica rigen condiciones especiales (véanse (44) y (57b)). 


\section{Las funciones discursivas de la cláusula con constituyente preverbal}

Como he señalado, la cláusula con verbo inicial permite sistemáticamente más de una lectura con relación a las constelaciones asertivas, sin que estas lecturas sean formalmente diferenciadas; dependen del contexto (sección 2.1.). Por ejemplo, (24a) y (24b) representan ambos una cláusula con verbo inicial. Sin embargo, en (24a) la expresión de participante lha nengnaykamka'aklha knaat 'nuestro lugar' forma parte de la presuposición y funciona como tópico, mientras que samtaj 'monstruo' en (24b) introduce un referente discursivo nuevo que, al no formar parte de lo presupuesto, está en foco (el adverbial yaamelket 'pozo' no afecta nuestra discusión aquí).

(24) a. Mook'alhma'a, yaame n-samk-ook

a.veces como F-mal-INT

lha neng-n-ayk-am-ka'a-klha knaat.

DEM:2 1PL-estar;sentarse-IND-EXT-SUBJ-ESP PP:PAS.REM

[El autor habla sobre su lugar de origen.] 'A veces, nuestro lugar (del que te hablo) estaba mal.'

b. V-a'aak samtaj yaamelket,

llegar;buscar-PRIM monstruo:F pozo:F

'Llegó un monstruo al pozo,'

c. yev-ey', n-ook hay', a-l-moop-eeyk-am'.

grande-IND igual-INT:L DEM:aquí F-DIST-blanco-IND-EXT

'era grande, así [como muestro], con rayas blancas.'

(Maangvayaam'ay', 25/6/2015)

A eso se añade otra observación: (24b) y (25b) no tienen tópico y expresan, por ende, un foco que se extiende sobre la cláusula entera. Sin embargo, a pesar de representar las mismas constelaciones asertivas, no pertenecen al mismo tipo de cláusula. Esta observación, que se repite con relación a todos los tipos de extensión que el foco puede tener, significa que las estructuras formales y las funciones pragmáticas no se relacionan de forma unívoca. De hecho, ambos tipos de cláusula no expresan solo la codificación pragmática de la información, sino que se usan en situaciones comunicativas diferentes y cumplen allí funciones discursivas específicas, las cuales se diferencian en la sección 4.1 con relación al foco estrecho, y en la sección 4.2 con relación al foco amplio. Las funciones discursivas del foco en la cláusula con verbo inicial (como samtaj en 
(24b)) complementan de forma heterogénea las funciones descritas y no se especifican en este trabajo.

(25) a. Ap-ta'h-engv-o-km-ek enlhet: M-volver-LOC-FACT-IBI-PRIM enlhet 'El enlhet volvió:'

b. "iSamtaj an-lheng-a-km-ok!" monstruo:F F-viajar-FACT-APR-SEC “"iSe acerca un monstruo!”’

(Haakok Aamay, 22/1/2007)

\subsection{El foco sobre un participante}

La siguiente clasificación de las funciones discursivas del foco sobre un participante (un foco estrecho) se basa en Dik (1981) y fue ampliada por mí donde ha sido necesario.

(a) Foco completivo (Dik 1981). De forma obligatoria, se usa la cláusula con constituyente preverbal si la expresión de participante llena un vacío en la información del receptor. Es frecuente que eso ocurra en la respuesta a una pregunta (26b).

(26) a. “¿Sok lhaa k-tahany-a'a ye?”, nae p-t-eem-ak ¿qué? PP:recién F-encontrar-SUBJ INTER PP:PAS.REM M-CÓP-IND-SEC maata'a pook. $\mathrm{PP}$ :siempre otro:M ““¿Qué encontraste?”, preguntó uno tras otro.'

b. " $i A ! ~ K e l v a a n a ' a ~ l h k a a$ k-tahany-a'ak, n-oo INJ mujer PP:recién 1SG-encontrar-SEC igual-INT:L nak antaava."

PP:PRES poroto.del.monte:F

““iAh! Me encontré con una mujer que era como el [arbusto de] poroto de monte."

c. “Antaava askok lho la $\underline{\underline{\text { la }}}$ n-tahany-a'ak poroto.del.monte:F F:bicho:F PP:INTER PP:duda F-encontrar-SEC lha", DEM:2

“'Supongo que vio al dueño del poroto de monte”,' 
t-eem-ak pook.

CÓP-IND-SEC otro:M

'supuso otro.'

(Ramón Ortiz, 8/8/2005)

(b) Foco de insistencia. Se usa la cláusula con constituyente preverbal para repetir información que el receptor no captó. Según este uso, se presenta el evento como presupuesto, insinuando un vacío de información del receptor que se refiere al participante (véase también sección 4.3).

(27) a. “Tep-kek pelten'.”

salir-PRIM luna:M

“"Salió la luna.",

b. “¿Haa?”

¿qué?

““¿Cómo?”,

c. “iPelten' ap-teep-ak!”

luna:M M-salir-SEC

““¡Salió la luna!””

(c) Foco de verificación. De forma obligatoria, se usa la cláusula con constituyente preverbal para verificar una pieza de información. Concretamente, el foco de verificación esta usado en preguntas que tienen la finalidad de comprobar si el participante preverbal se relaciona de la manera indicada con el evento expresado.

(28) “YYoonges! ¿Tata’a ya apk-el-laap-ak?”, ap-tamh-ak lengko. muchachos gallina:F PP:INTER M-DIST:L-matar-SEC M-CÓP-SEC menonita ““¡Muchachos! ¿Están matando las gallinas?”, gritó el menonita.’ (Metyeeyam', 3/2004)

(29) ¿Lheep ya apk-engk-ak yavhan ah-ak sek-pongkan-m-a? 2SG:M PP:INTER M-cortar-SEC miel:M 1SG-POS 1SG-eligir-IND-INF ¿¿Eras vos quien abrió la colmena que yo había elegido?'

(Haakok Aamay, 31/10/1999)

(d) Foco de precisión. Como señalado en la sección 1, el enlhet no ofrece ningún mecanismo sintáctico para diferenciar entre el primer y el segundo participante. Este obstáculo se contrarresta parcialmente presentando un suceso a través de una cláusula con verbo monovalente (30a), que está 
seguida por otra con un verbo bivalente del mismo valor semántico (30b) y sirve para identificar al actor del suceso a través del constituyente preverbal. Para el desarrollo temático, dicho actor es de importancia secundaria, lo que indica que la función de este foco es precisar el evento reportado, no la de introducir un referente que en el discurso subsiguiente serviría de tópico.

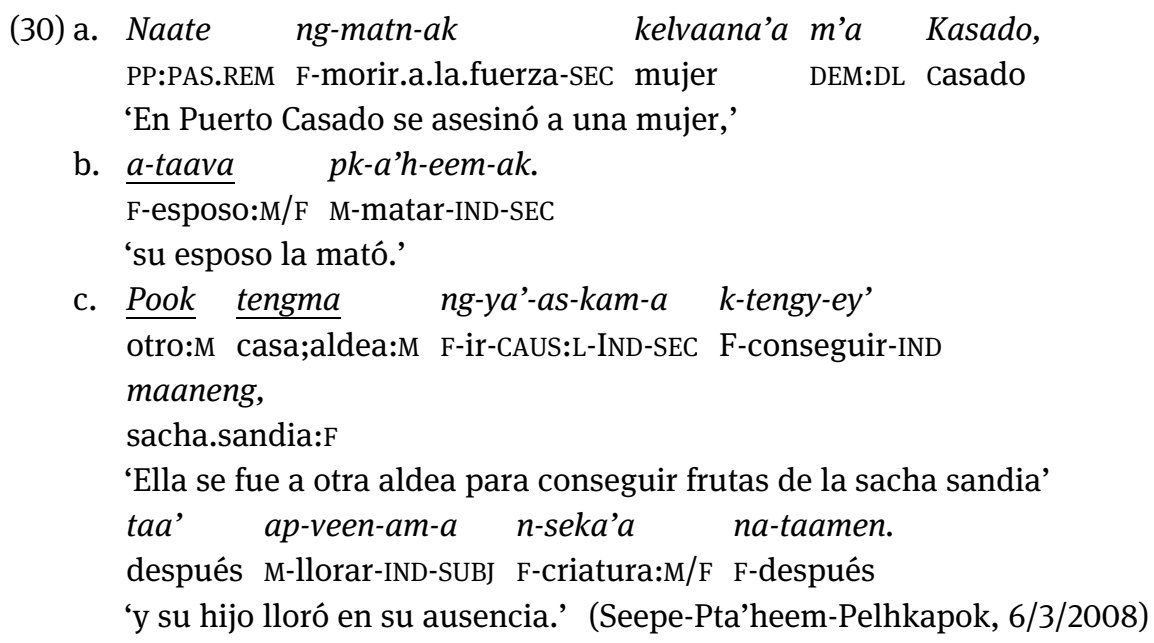

Paralelamente, este foco se emplea para precisar información sobre un referente dado ((31) a (33)).

(31) Taan sek-lho-y'-a mook s-enamt-eem, después 1SG-recibir-IND-SUBJ otro:F 1SG-subir-IND 'Después recibí otro medio,' meyoo-ya-m'-neeten naat ak-lho-k'ak. ir-IND-INF-arriba PP:PAS.REM 1SG-recibir-SEC 'recibí un avión.’ ～(Maangvayaam’ay', 20/12/2012)

(32) Seklha lhne k-yepho-ngv-a'a, na-v'-oo raahek inmediatamente PP:CIT F-aparecer-LOC:L-SUBJ F-punta:F-INT F:nariz:F ne ng-yepho-ngv-esk-a'ak, apk-enyah-a. PP:CIT F-aparecer-LOC:L-CAUS-SEC M-correr;huir-SUBJ 'Apenas apareció [la víbora] - hizo aparecer la punta de su nariz-, él se dio a la fuga.'

(Maangvayaam'ay', 20/12/2012) 
(33) $Y a^{\prime} v-a e-k m-e$ nnekhe tav-akp-ok ma'a yaata'ay'. querer-IND-APR:L-PRIM PP:FRUST M:comer-PASIVO:M-POT DEM:DL cabra:M 'Pretendían comer las cabras.' Tekyav-a nnekhe', morir.a.la.fuerza:DIST-SUBJ PP:FRUST '[Algunas] fueron carneadas.' Apk-aanet yaata'ay' ap-tekyav-ak. M-dos cabra:M M-morir.a.la.fuerza:DIST-SEC 'Dos cabras fueron carneadas.'

(Kam’aatkok Ketsek, 10/1/2007)

(e) Foco de sustitución (Dik 1981). De forma obligatoria, se emplea el constituyente preverbal para corregir la suposición de una participación de cierto referente en el evento expresado.

(34) a. “Lheng-ke nlho seta’” viajar-PRIM PP:INTER 1SG:abuelo “"¿Mi abuelo se fue?”,

b. 'Paej, haave', taata lhkaa p-lheng-ak. NEG no.es 1SG:padre PP:recién M-viajar-SEC ““No, no era él; se fue papá.”,

(35) a. Taa' apk-el-ta'h-ae-klh-o a neeten, después M-DIST-volver-IND-REP-SUBJ DEM:DL arriba 'Después volvieron hacia arriba'
b. l-oom-oo pk-el-yav'aklhoho, haave p-hangaok; seguir-IND-INT M-DIST-todo;entero;en.cuerpo no.es M-alma:F 'en sus cuerpos, no como almas'
c. vaamlha pk-el-yav'aklhoho pk-el-mey-ae-klh-ak neeten. solo M-DIST-todo;entero;en.cuerpo M-DIST-ir-IND-REP-SEC arriba 'en sus cuerpos se fueron para arriba.' (Maangvayaam'ay', 1/5/2001)

La pieza de información corregida no está necesariamente explícita; en (36c), yaapa 'mosca' sustituye tácitamente al golpe de cuchillo de (36a).

(36) a. Naa m-pa'lh-am-ak as'e, savov moy-engk-eem, PP:PAS.REM F-romperse-IND-SEC DEM:aquí cuchillo:F IMP-cortar-IND 'Se le rompió esta parte [de su cuerpo], alguien le dio un golpe con un cuchillo,' 
b. paej ke ngy-etsep-m-ak.

NEG PP:pero M-morir-IND-SEC

'pero no murió.'

c. Yaapa yka pk-a'h-eem-ak

mosca:M PP:pero M-matar-IND-SEC

'Sin embargo, eran las moscas que lo mataron'

ap-v-a'ae-kt-amo ha lengko.

M-llegar;buscar-IND-VENT-SUBJ DEM:aquí menonita

'cuando vino junto a los menonitas.'? (Metyeeyam', 8/8/2006)

En (36), “matar" incluye "morir” y es, por ello, usado como un evento presupuesto. Indica, de esta manera, que los criterios para asumir un evento como presupuesto no se limitan a una identidad lexical como en (14). En (37), incluso, “defender" es un evento opuesto a "matar"; no obstante, la relación temática creada con esta oposición es suficiente para entenderlo como presupuesto y plantear, así, un foco estrecho (de sustitución). Cabe añadir que, al usar aquí la cláusula con constituyente preverbal, el autor indica que opone un participante a otro, el “nosotros” al "padre”. Si optara en (37b) por aquella con predicado verbal inicial, lo que también sería posible, opondría un evento a otro, el "defender” al "matar”.

(37) a. Alkoove, mah-ay' yekhe ngy-a'h-e p-yaap.

Alkoove querer-IND PP:FRUST M-matar-POT M-padre

'A Alkoove, su padre lo quería matar.'

b. Nengko'o yka neng-mey-aok taata nnenga, meeme

1PL PP:pero 1PL-defender-SEC 1SG:padre finado 1SG:madre nnenga.

finado

'Pero nosotros lo evitamos, yo, mi finado padre y mi finada madre.'

(Seepe-Pta'heem-Pelhkapok, 7/2/2006)

(f) Foco de restricción (Dik 1981). De forma obligatoria, se usa la cláusula con constituyente preverbal para indicar que el desarrollo del evento expresado queda restringido al participante preverbal.

(38) a. Ka' nen-lho-k'a anmen taata nnenga alhta, (...) después 1PL-recibir-SUBJ chicha:F 1SG:padre finado PP:PAS 'Después, mi finado padre recibió chicha;' (. .) 
b. vaamlha tres apk-et-lho-k'ak, p-yen-ayk-aok yo'yaam' solo tres M-DIST-recibir-SEC M-beber-IND-COMP para e-tengy-amk-ok ma'a yengmen. M-conseguir-COMP-POT DEM:DL agua:F 'Solo tres recibieron, la tomaron para conseguir lluvia.'

(Seepe-Pta'heem-Pelhkapok, 6/2/2005)

(39) M-ong-vank-ehek nahan ang-mokt-ak ma'a a'sok na-vhak, NEG-1PL-poder-NO.FACT PP:COOR 1PL-tirar-POT DEM:DL cosa:F F-silvestre 'Era imposible cazar animales,' teem valay, ya-halhn-amk-ok ay-enmong-aam. pues paraguayo M-escuchar-COMP-POT F-estallar-IND 'pues los paraguayos escucharían los estallidos.' Vaamlha yavhan eeka' ap-t-oomm-akp-ok; solo miel:M PP:pero M-comer-IND-PASIVO:M-SEC 'Se comía solo miel;' naate n-t-eem-ak meeme ka-ltemn-am'. PP:PAS.REM F-CÓP-IND-SEC 1SG:madre F-relatar-EXT 'asî lo contaba mi madre.'

(Maangvayaam'ay', 20/1/2007)

(g) Foco de selección o identificación (Dik 1981). Se usa la cláusula con constituyente preverbal para seleccionar o identificar un participante entre varios referentes accesibles.

(40) Ko'o saat a-lk-ok e-mamye’.

1SG PP:FUT 1SG-sacar-POT 1SG-primero

'Yo voy a quitarlo primero.'

E-vatnap lha payh-oo nak. Alhaaye saat lheep. M-soplar DEM:2 estar.cerca-INT PP:PRES más.tarde PP:FUT 2SG:M 'Soplá vos ahí. Después te va a tocar a vos.'

(Seepe-Pta'heem-Pelhkapok, 6/2/2005)

El contraste insinuado por el foco de selección puede quedar implícito. En (41) se genera entre los hombres, que son tácitamente incluidos en aptengyeeykaok na.lhyeenep 'cazar venados en la isla de monte', y kelvaana'a 'las mujeres', que son explícitamente mencionadas.

(41) Kel-vesk-a'a naak nen-taamen, ak-talhnaam' e-l-pa'met-am', DIST-líder-PL PP:PAS.REM 1PL-detrás F-tarde:F M-DIST-hablar-EXT 'Los líderes de antes conversaban a la tarde,' 


\begin{abstract}
e-l-lheen-ek ap-tengy-eeyk-aok na lhyeenep. M-DIST-decir-POT M-conseguir-IND-COMP PP:PRES montecito:F 'hablaban de la batida en la isla de monte.' “iKelvaana'a saat ka-tengy-amk-ok peheya', mujer PP:FUT F-conseguir-COMP-POT batata:F “'Las mujeres van a preparar las batatas' neng-engkah-ayk-aok yengmenaata popyet!” e-tn-ehek. 1PL-juntar-IND-COMP caldo:F venado:M M-CÓP-POT 'que se comerán con el caldo de venado”, solían decir.'
\end{abstract}

(Metyeeyam', 24/5/2002)

(h) Foco de expansión (Dik 1981). Se usa la cláusula con constituyente preverbal para extender un evento a otro participante más. Nótese que apkapok 'su espalda' en (42a) y (42b) refieren a entidades diferentes; por lo tanto, el referente de apkapok 'su espalda' en (42b) no forma parte de la presuposición.

(42) a. Taan, melask-emm-alhk-eek nek aapetek ma'a, después repartir-IND-PASIVO-PRIM PP:CIT F:carne:F DEM:DL 'Después repartieron la carne' p-nengkan-m-a popyet a apk-apok. M-poner:DIST-IND-INF venado:M DEM:DL M-espalda:F 'y el venado se la puso sobre la espalda.'

b. Apk-apok naha' a-pakan-m-ak lhaapen. M-espalda:F PP:COOR M-poner-IND-SEC ñandú:M 'El ñandú se la colgó también sobre la espalda.'

(Maangvayaam'ay', 1/5/2001)

\title{
4.2 El foco oracional y el foco compuesto
}

En esta sección se analizan las funciones discursivas del foco oracional y el foco compuesto (focos amplios), que se superponen parcialmente. Respecto a su clasificación sigo la terminología de Sasse (1995), ampliándola donde es necesario.

(a) Foco anunciativo (Sasse 1995, 14). Se usa la cláusula con constituyente preverbal en respuestas que no contienen información presupuesta - que no son aserciones sobre un referente- y carecen, por ello, de tópico (véase también (17)). Son respuestas a preguntas que se refieren en un sentido amplio a una situación, como (43) y (44). El foco anunciativo, que se combina tanto con verbos monovalentes (43) como bivalentes (44), siempre es un foco sobre la cláusula entera. 
(43) “¿So p-tengy-a' ye?” ¿qué? M-conseguir-SUBJ INTER “¿Qué buscas?”

"iMayka'a lhkaa p-v-a'ak aha'e tengma!" visitante PP:recién M-llegar;buscar-SEC DEM:3 casa;aldea:M “"iLlegó un visitante a la aldea!”'

(Seepe-Lhama, en Unruh y Kalisch 2000, 97)

(44) “LLha' ak-tamh-a yeee?”

PP:CONJ F-CÓP-SUBJ INTER

“¿QQué pasa?”

“iMaha lhkaak e-l-yoot-’ak!”

avispa:F PP:recién INV.SG-DIST:L-tirar-SEC

““iMe picaron avispas!”’ (Seepe-Lhama, en Unruh y Kalisch 2000, 96)

El foco anunciativo no está limitado a las respuestas, sino que se usa de manera general para expresar una proposición que tiene, desde la perspectiva de quien habla, un nexo con la situación inmediata en cuanto resalta que es inesperada e insinúa sorpresa o implica una alerta (véanse también (18) a (20)).

(45) Neng-vet-'aak naate yke nhan nengko'o k-teyapma, 1PL-ver-PRIM PP:PAS.REM PP:pero PP:COOR 1PL F-estrella.fugaz:F 'Vimos [en aquella ocasión] una estrella fugaz,' mey-a-klh-o a; ka-lhnaan-em-s-ehe nak ir-FACT-REP-SUBJ DEM:DL F-perderse-APR:L-CAUS-NO.FACT PP:PRES nahan ma'a. “¡Ap-yoholhma pk-etsap-ak!”, tamh-ak amya'a. PP:COOR DEM:DL M-anciano M-morir-SEC CÓP-SEC noticia:F 'se fue a aquel lado; ella no lo ignora: “Murió un anciano!”, se comentó.'

(Seepe-Pta'heem-Pelhkapok, 6/3/2008)

(46) Mey-a-klh-eek amya'a ahay'e taata: ir-FACT-REP-PRIM noticia:F DEM:3 1SG:padre 'La noticia llegó a lo de papá:' "iNengmaskeem an-lheng-a-km-ok aha'e!" enfermedad:F F-viajar-FACT-APR-SEC DEM:3 ““¡Allí viene la enfermedad!” (Metyeeyam', 24/5/2002)

En cambio, en (47b) el evento está esperado y le falta el elemento de sorpresa; en consecuencia, no se emplea la cláusula con constituyente preverbal. Por la 
misma razón, tampoco se emplea en (27a) Tepkek pelten' 'Salió la luna'. Paralelamente, (18a) con verbo inicial (Teyakmek yaamkentem'aahak apak 'Se cayó tu bolsa') transmite una simple información; si la misma proposición fuese expresada con el empleo de un constituyente preverbal implicaría una alerta a la que se esperaría que el interlocutor reaccione.

(47) a. “A-lhong saat ko'o, a-nempa'vo-m-ok saat, '“Salgo, voy a cazar,'

a-tengy-ak saat ko'o aapetek, lhaapen”, ap-tamh-ak 1SG-conseguir-POT PP:FUT 1SG F:carne:F ñandú:M M-CÓP-SEC taata.

1SG:padre

'voy a conseguir carne, ñandú”, dijo papá'

Na'sook nahan, ang-ely-ohok maata'a ap-ya'-s-a-klha'. de.verdad PP:COOR 1PL-mirar-INT:POT PP:siempre M-ir-CAUS:L-SUBJ-ESP 'Así ocurrió y nosotros miramos hacia dónde había ido.' Paej $e$-v-'a-kt-a', vaamlha talhnaam' NEG M-llegar;buscar-FACT-VENT-SEC solo tarde:F ap-v-a'ae-kt-a', e-patm-ohok lhaapen. (...) M-llegar;buscar-IND-VENT-INF M-alzar-INT:POT ñandú:M $V a^{\prime}$ :

INJ:atención

'No volvió, recién a la tarde solía llegar, cargado con un ñandú. De repente:'

b. “Ap-v-a'a-kt-aak taata, meeme. M-llegar;buscar-FACT-VENT-PRIM 1SG:padre 1SG:madre “'Mamá, papá viene.'

c. ¡Ang-elemyelh-amk-ok!", tamh-ak ko'o maata'a. 1PL-ir.a.encontrar-COMP-POT CÓP-SEC 1SG PP:siempre ‘¡Vamos a encontrarlo!”, solía yo decir.'

(Maeklha'ay'-Pya'yeem, 7/2/2011)

(b) Foco explicativo (Sasse 1995, 18). Tanto el foco oracional como el foco compuesto son usados para presentar información que explica la proposición previa (véase también (21)).

(48) K-el-pekh-ek'aak enlhet, F/M-DIST-apurado-PRIM enlhet 'La gente está apurada,' 
$\frac{\text { mayka'a }}{\text { visitante }} \frac{\text { lhnek }}{\text { PP:CIT }} \frac{\text { lhaak }}{\text { PP:recién FUT M:DIST-LLEGAR;BUSCAR-POT }}$ l- $v$ - $a$ '.
'dicen que van a llegar visitantes.'

Se responde con un foco explicativo a consultas sobre una situación, si la respuesta no implica ningún nexo con la situación inmediata y le falta ese elemento de sorpresa o alerta que caracteriza el foco anunciativo. En (49b) aparece el foco explicativo como foco oracional; en (49c) y (49d) como foco compuesto.

(49) a. “¿Naat lha' ap-tengy-ey'-a sevhen ke?” PP:PAS.REM PP:CONJ M-conseguir-IND-SUBJ nivaclé INTER ““Qué era lo que buscaron los nivaclé?””

b. "Noolheng ne p-menyelh-m-ak enlhet ma'a; caballo:M PP:CIT M-robar-IND-SEC enlhet DEM:DL “'En aquella ocasión, los enlhet habían robado caballos;'

c. kempokhakme' p-lheng-aaya-km-ok. guerreros:M M-viajar-IND-APR-SEC 'vinieron como guerreros,'

d. enlhet yekhe p-maagah-ay'-ak e-l-naap-ok ma'a." enlhet PP:FRUST M-querer-IND-SEC M-DIST:L-matar-POT DEM:DL 'pretendían matar a los enlhet.”'

(Metyeeyam', 24/5/2002)

(50) “ ¿Lha' ap-ya'-s-a taata?”

PP:CONJ M-ir-CAUS:L-SUBJ 1SG:padre

““¿A dónde se fue papá?””

“Yaata'ay' lhaa $\quad$ pakh-engv-ee-klh-a.”
cabra:M PP:recién M:buscar-LOC-FACT-PRV-SEC
“"Se fue a buscar las cabras.”"

(c) Foco elaborativo. El foco compuesto es usado para presentar información sobre un referente discursivo activado, la cual no será retomada después. Esta función discursiva es similar a la del foco de precisión que, sin embargo, es un foco estrecho, ya que en su caso el evento forma parte de lo presupuesto.
(51) Tekyav-am-ke' 'sa pk-etk-ek. morir.a.la.fuerza:DIST-IND-PRIM PP:nomás M-hijo:M/F-PL 'Sus hijos fueron asesinados sin nada más.' 


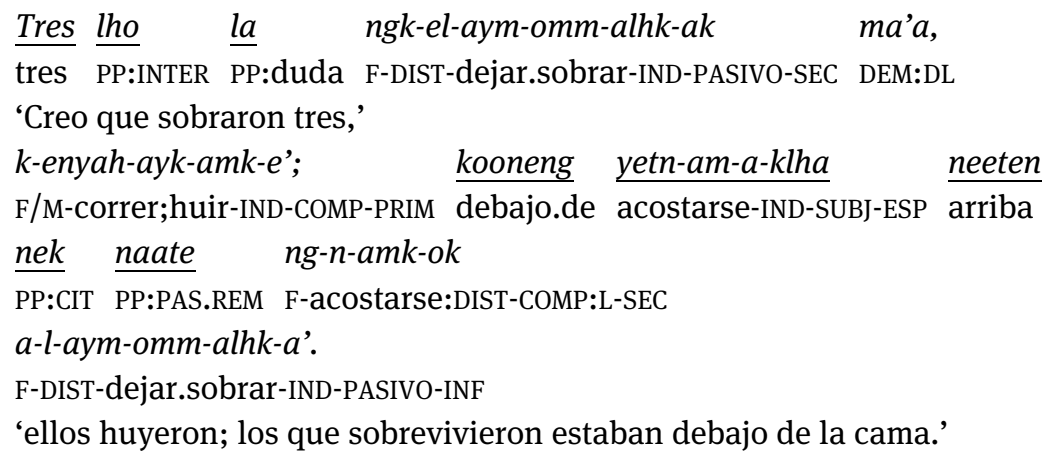

(Sa'ko-Yenyeem, 20/9/2013)

(d) Foco de trasfondo (“episode opening” de Sasse 1995, 16). El foco oracional (52a) y el foco compuesto (52b) se usan para presentar un contexto o un trasfondo temático. Aunque es posible, como en (52a), que uno de los referentes de este trasfondo se vuelva el tópico de la cláusula siguiente ((52b)), no se trata de una estrategia para introducir nuevos referentes.

(52) a. Yavhan ap-tengy-eyk-amk-ok enlhet, miel:M M-conseguir-IND-COMP-SEC enlhet '[En esa ocasión] estaban consiguiendo miel,'

b. lengko pk-emp-ayk-amk-ok, nalhma pk-el-ya'a-sk-am-ak, menonita m-venir.de-IND-COMP-SEC monte:F M-DIST-ir-CAUS:L-IND-SEC teyaanyam'.

cazería:F

'salieron de lo de los menonitas, se fueron al monte para cazar.'

c. Taa' p-tengy-eyk-amk-o yavhan, después M-conseguir-IND-COMP-SUBJ miel:M 'Entonces, cuando iban a conseguir miel,' taa' apk-elh-ayke-klh-o nep-taan apk-elnan-talhn-am-a, después M-tirar:DIST-IND-REP-SUBJ M-detrás M-DIST-entrar-IND-INF 'dejaron atrás su ropa,' lengko pk-elnan-talhn-am-a, menonita M-DIST-entrar-IND-INF 'la ropa de (los) menonitas,' pehevee-klh-oo kham apk-elnan-talhn-am-a pk-el-ya'vat-eem. en.cambio-REP-SUBJ PP:REP M-DIST-entrar-IND-INF M-DIST-corto-IND 'y se pusieron otra vez su taparrabo.'

(Sa'ko-Yenyeem, 14/8/2005) 
(e) Foco de conclusión. Se usa la cláusula con constituyente preverbal para repetir un evento en forma de una conclusión, expresando información presupuesta como si no lo fuera. Esta contradicción a las condiciones asertivas reales está empleada para relegar aquello que se acaba de describir a una información de trasfondo (53b) o para cerrar un tema (54b).

(53) a. Naa y-emp-a'ak hay', Yey'keenaateng, PP:PAS.REM 1SG-venir.de-SEC DEM:aquí Yey'keenaateng [Llegué solo a la casa de ella.] 'Vine de Yey'keenaateng,' neng-el-ya'-s-a ng-yaanmaan, hayyy. 1PL-DIST-ir-CAUS:L-SUBJ F-iniciación:F INJ 'y nos fuimos a una fiesta de iniciación.'

b. Yaanmaan naak n-el-ya'a-sk-am-ak. iniciación:F PP:PAS.REM 1PL-DIST-ir-CAUS:L-IND-SEC 'Nos fuimos a una fiesta de iniciación.' [Sigue la descripción del encuentro con la mujer.]

(Metyeeyam', 7/2003)

(54) a. Pang, ap-nek nak a, e-lyetsamong, taa' cactus:M M-harina:F PP:PRES DEM:DL M-exprimir-POT después e-lk-o p-me-ok. Sas naate m'a pang M-sacar-POT M-mano:F-PL lindo PP:PAS.REM DEM:DL cactus:M ap-yengmenek;

M-líquido:F

'Exprimían el cactus, los pedazos de cactus, y se lavaban las manos. El jugo del cactus era bueno;'

b. pang yekhe lk-o p-me-ok. cactus:M PP:FRUST M:sacar-POT M-mano:F-PL 'con el cactus se lavaban las manos.'

(Yamasma’ay', 7/8/2007)

\subsection{Una lectura alternativa de la posición inicial}

En 3.3 he presentado una clasificación de las extensiones sintácticas del foco en las cláusulas con constituyente preverbal, que se basa en una distinción de las condiciones asertivas bajo las cuales el foco es usado. Ahora bien, hay ciertos elementos formales que pueden acompañar un foco estrecho; por ejemplo, la partícula eeka' $\sim e e k a \sim k e$ 'pero' (en (36c) y (37b)), que tiene entre sus funciones la de marcar contraste. Sin embargo, indicadores de esta índole no son necesarios ni suficientes para hablar de un foco estrecho. En otras palabras, la 
extensión de un foco en una cláusula específica no puede ser determinada exclusivamente a partir de criterios formales; o sea, la distinción formal entre foco estrecho y foco amplio es vaga o subdiferenciada.

Eso plantea una dificultad, porque las condiciones asertivas en una cláusula concreta no son siempre totalmente claras, ya que las opciones lexicales para denotar un mismo evento son múltiples, a la vez que las pautas cognitivas que evocan eventos relacionados al tema discursivo son complejas (sección 4.1). Entonces, en la medida en que el límite entre aquello que no es recuperable del contexto y aquello que es semántica, contextual o situacionalmente evocado no es discreto, tampoco es posible determinar de forma inequívoca si el evento denotado por el verbo está presupuesto o no y, por ende, especificar la extensión del foco en cuestión. Por ejemplo, en (55b) hablaríamos de un foco de sustitución (que es un foco estrecho), aunque, en un sentido estricto, el evento no está presupuesto; está evocado por el contraste entre "querer" y "ser juntado por obligación” (véase lo señalado con relación a (37)).

(55) a. Haave k'o sek-maayah-ay' lha, no.es 1SG 1SG-querer-IND DEM:2

'No era yo quien lo quería,'

b. apk-el-yapko-ho knaate $\underline{\text { kka }}$ e-ypet-s-eeyk-am'. M-DIST-tío.materno-PL PP:PAS.REM PP:pero INV.SG-juntarse-CAUS-IND-EXT 'sus tíos me juntaron con él.' (Kam'aatkok Ketsek, 8/1/2008)

En un enunciado como (56b), las constelaciones son más complejas aún, porque el mismo permite dos lecturas con relación a la extensión del foco. La pregunta de (56a) fue dirigida a una persona que lleva pomelos; sirve como saludo. Por eso, se puede interpretar la respuesta de (56b) como una aserción sobre una situación entera, como un foco compuesto (foco explicativo). Sin embargo, el foco en la respuesta se puede entender también como un foco estrecho (un foco completivo), si se asume que "ir" es temáticamente evocado por el verbo "llevar", de manera que el predicado está presupuesto.

(56) a. “¿Soolyaatektama yla patm-ak?”

pomelo:F PP:INTER M:alzar-SEC

““¿Llevás pomelos?””

b. "Eehe, Menlhanmaklha paa ngka-l-ya'a-ks-ek." sí Menlhanmaklha' FUT F-DIST-ir-CAUS:L-POT “"Sí, irán a Menlhanmaklha.”, 
Tal ambigüedad, que surge del hecho de que las diferentes extensiones sintácticas del foco no están diferenciadas formalmente, se manifiesta en una cantidad considerable de casos (en realidad, varios de los enunciados citados en este trabajo podrían discutirse con relación a lecturas alternativas). En (57b), el evento “comer” no está presupuesto, pero la presencia explícita del patrón lo evoca, pues era común que los patrones menonitas sedujeran a sus empleados con buena comida. Por lo tanto, se puede leer (57b) bien como un foco de selección (un foco estrecho), bien como un foco anunciativo (un foco amplio).

(57) a. Nen-teyaap-eya-km-ook naat ang-elan-am',

1PL-no.dormir-IND-APR:L-INT PP:PAS.REM 1PL-observar-EXT

[Se habla de la cocción de ladrillos]. 'No dormíamos mientras los cuidábamos,'

paej nen-teyan-m-a.

NEG 1PL-dormir-IND-INF

'no se dormía.'

b. “iAapetek saate tav-amk-ok!”, t-eem-ak maata'a

$\mathrm{F}$ :carne:F PP:FUT M:comer-COMP-POT CÓP-IND-SEC PP:siempre

lengko. (...)

menonita

““¡Van a comer carne!”, solía decir el menonita. (...)’

c. Taa' ak-ya-ngv-ae-km-oo ak-maases

después F-grande-LOC-IND-IBI-INT F-rico

neng-el-tamh-ayk-am', mepke teyen'.

1PL-DIST-trabajar-IND-EXT:L sin sueño:F

'Después aumentaron nuestras ganas de trabajar, sin [sentir el] sueño.'

(Savhongvay', en Unruh y Kalisch 2000, 238)

Esta ambigüedad es aprovechada sistemáticamente por los hablantes, conjugando las dos lecturas posibles de la siguiente manera: aunque una cláusula como (57b) puede entenderse como la presentación de una proposición entera (como un foco amplio), la insinuación de un marco situacional particular hace que la información relevante caiga sobre el referente aapetek 'la carne', mientras que el evento es relativamente de poca importancia; podría ser, también, por ejemplo, cocinar, comer, recibir o llevar a casa. Identificamos, así, otra función de la posición preverbal, la asociativa. Sirve para presentar un referente en un marco proposicional que es de importancia secundaria, porque la relación del referente con el tema discursivo no importa más allá de una vaga evocación. Probablemente, el foco de insistencia deba entenderse en realidad como un constituyente preverbal con esta función asociativa. 


\section{Conclusión}

Para codificar la estructura informativa, el enlhet se vale de una distinción de dos tipos de cláusula, una con verbo inicial, que sirve típicamente para afirmar información sobre un referente presupuesto (en otra terminología, tiene una partición informativa entre tópico y comentario; Lambrecht 1994, 126); y otra con participante inicial, a través de la cual se expresa información que no está presupuesta, o sea un foco. En el precedente análisis, se han identificado las posibles extensiones sintácticas de este foco, relacionándolas con diversas funciones discursivas, tal como se resumen en la Tabla 1.

Tabla 1: Tipos de foco en el enlhet.

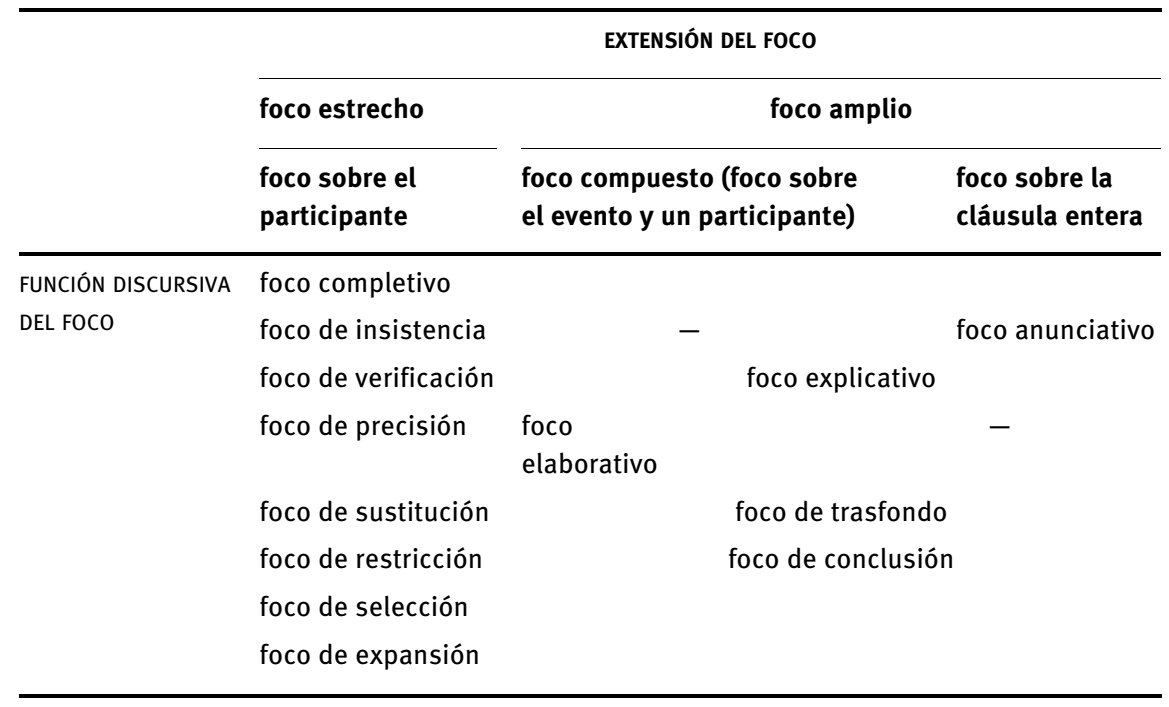

El foco estrecho y el foco amplio difieren con relación a su alcance sobre constituyentes sintácticos, son usados bajo condiciones asertivas distintas y tienen funciones discursivas desiguales. A pesar de esta discrepancia, ambos tipos de foco son expresados empleando una misma posición sintáctica, la preverbal. Esta discrepancia impide considerar tal empleo como un hecho natural; debe ser explicado: es posible aprovechar una sola posición sintáctica para expresar los diferentes tipos de foco, porque los participantes que la ocupan comparten la característica de ser un no-tópico. En realidad, aunque no haya sido un tema 
de este texto, la posición preverbal está usada sistemáticamente para expresar no-tópicos; por ejemplo, cierto tipo de adverbiales.

Mientras la distinción entre foco estrecho y foco amplio es vaga o subdiferenciada, el foco oracional y el foco compuesto tienen una distribución complementaria, que está definida por la cantidad de expresiones de participante en la cláusula correspondiente. Al mismo tiempo, sus funciones discursivas se superponen parcialmente. Por ambas razones, el foco compuesto no es un subgrupo del foco oracional.

En vistas a futuras investigaciones, se debe resaltar que el constituyente preverbal no se limita a expresiones de participante, y que una mejor comprensión del mismo debería examinar la gama completa de usos. Tal análisis debe incluir también la frecuente discontinuidad de una misma expresión de participante, apareciendo una parte en la posición preverbal y otra detrás del verbo. Además, debe considerarse que la posición preverbal es sensible a varios y heterogéneos parámetros semánticos y sintácticos que requieren o prohíben su uso. Por último, las seis lenguas de la familia enlhet-enenlhet se comportan de manera similar con relación a los fenómenos descritos, aunque muestran diferencias en los detalles. Para una mayor comprensión de los tipos de cláusula en estas lenguas - también de su dimensión histórica-, un análisis de las diferencias entre ellas sería altamente esclarecedor.

\section{Agradecimientos}

Agradezco a Andrés Salanova, Mily Crevels, Valeria Belloro y dos revisores anónimos sus comentarios a una versión previa de este texto.

\section{Referencias}

Ashby, William y Paola Bentivoglio. 1993. Preferred argument structure in spoken French and Spanish. Language Variation and Change 5. 61-76.

Chafe, Wallace. 1987. Cognitive constraints on information flow. En Rusell Tomlin (ed.). Coherence and grounding in discourse, 21-51. Amsterdam: John Benjamins.

Dik, Simon. 1981. On the typology of Focus Phenomena. En Teun Hoekstra, Harry van der Hulst y Michael Moortgat (eds.). Perspectives on Functional Grammar, 41-74. Dordrecht: Foris.

Fabre, Alain. 2005. Los pueblos del Gran Chaco y sus lenguas, primera parte: Los enlhetenenlhet del Chaco Paraguayo. Suplemento Antropológico 40(1). 503-569. En línea http://www.academia.edu/3611583/Dic_Enlhet_Enenlhet [25/4/2019].

Hockett, Charles F. 1958. A Course in Modern Linguistics. Oxford: Macmillan. 
Kalisch, Hannes. 2009-2010. Los constituyentes de la cláusula enlhet (enlhet-enenlhet). Esbozo de una cláusula omnipredicativa. Amerindia 33/34. 109-150.

Karttunen, Lauri. 1976. Discourse Referents. En James Mc. Cawley (ed.). Notes from the linguistic underground, 363-385. New York: Academic Press.

Lambrecht, Knud. 1994. Information structure and sentence form. Topic, focus and the mental representations of discourse referents. Cambridge: Cambridge University Press.

Sasse, Hans-Jürgen. 1995. Theticity and VS order: a case study. Sprachtypologie und Universalienforschung (STUF) 48. 3-31.

Unruh, Ernesto y Hannes Kalisch. 2000. Ya'alva Pangcalhva. (Biblioteca Paraguaya de Antropología, vol. 33). Asunción y Ya’alve-Saanga: CEADUC y Nengvaanemkeskama Nempayvaam Enlhet.

Unruh, Ernesto y Hannes Kalisch. 2003. Enlhet-Enenlhet. Una familia lingüística chaqueña. Thule. Rivista italiana di studi americanistici 14/15. 207-231. 
